\title{
Pleomorphic Liposarcoma
}

National Cancer Institute

\section{Source}

National Cancer Institute. Pleomorphic Liposarcoma. NCI Thesaurus. Code C3705.

A liposarcoma characterized by the presence of varying proportions of pleomorphic lipoblasts in a background that resembles undifferentiated pleomorphic sarcoma. It is the rarest liposarcoma variant and usually has an aggressive clinical course. 